\title{
On Ray Shooting in Convex Polytopes*
}

\author{
Jiří Matoušek ${ }^{1}$ and Otfried Schwarzkopf ${ }^{2}$ \\ ${ }^{1}$ Katedra aplikované matematiky, Universita Karlova, Malostranské nám. 25, \\ 11800 Praha 1, Czech Republic, and \\ Institut für Informatik, Freie Universität Berlin, Arnimallee 2-6, \\ 1000 Berlin 33, Federal Republic of Germany \\ matousek@cspguk11.bitnet \\ ${ }^{2}$ Universiteit Utrecht, Vakgroep Informatica, Postbus 80-089, \\ 3508 TB Utrecht, The Netherlands \\ otfried@cs.ruu.nl
}

\begin{abstract}
Let $\mathscr{P}$ be a convex polytope with $n$ facets in the Euclidean space of a (small) fixed dimension $d$. We consider the membership problem for $\mathscr{P}$ (given a query point, decide whether it lies in $\mathscr{P}$ ) and the ray shooting problem in $\mathscr{P}$ (given a query ray originating inside $\mathscr{P}$, determine the first facet of $\mathscr{P}$ hit by it). It was shown in [AM2] that a data structure for the membership problem satisfying certain mild assumptions can also be used for the ray shooting problem, with a logarithmic overhead in query time. Here we show that some specific data structures for the membership problem can be used for ray shooting in a more direct way, reducing the overhead in the query time and eliminating the use of parametric search.

We also describe an improved static solution for the membership problem, approaching the conjectured lower bounds more tightly.
\end{abstract}

\section{Introduction}

Let $d$ be a fixed (small) integer, and let $\mathscr{P}$ be a convex polytope in $\mathbb{E}^{d}$ with at most $n$ facets, i.e., an intersection of $n$ half-spaces. We consider the following algorithmic problems for $\mathscr{P}$, listed in the order of increasing generality:

\footnotetext{
* Part of the work on this paper by Jiři Matoušek was supported by Humboldt Forschungsstipendium. Otfried Schwarzkopf acknowledges support by the ESPRIT II Basic Research Action of the European Community under Contract No. 3075 (project ALCOM). This research was done while he was employed at Freie Universität Berlin. Furthermore, part of this research was done while he visited INRIA-Sophia Antipolis.
} 
- The membership problem. We want to build some data structure (storing some information about $\mathscr{P}$ ), such that, for a given query point, we can quickly decide whether it lies in $\mathscr{P}$.

- The membership with witness problem. This is as the membership problem, but if the answer to the query is NO (the query point lies outside $\mathscr{P}$ ), we require that one of the $n$ half-spaces defining $\mathscr{P}$ is returned, which does not contain the query point.

- The ray shooting problem. In this case the query is specified by a ray $\rho$, originating inside $\mathscr{P}$, and the goal is to find the first facet of $\mathscr{P}$ crossed by the ray. ${ }^{1}$

The membership problem has been intensively studied in computational geometry (mainly in dimensions two and three), and it falls into the area known as point location problems. From another perspective, this problem can be viewed as belonging to range searching, and, indeed, the known efficient solutions for this membership problem come from research in range searching (at least those for dimension $d \geq 4$ ). The point location and range searching problems are of some interest for direct practical applications, but they are still more significant as subroutines used in other problems.

Our main goal is to show that certain data structures for the membership problem for $\mathscr{P}$ can also be used (perhaps slightly augmented) for ray shooting in $\mathscr{P}$. Ray shooting problems (which can also be formulated in many different settings) are also quite significant, e.g., they play an important role in various hidden surface removal algorithms and related problems directly motivated by computer graphics. Ray shooting in a convex polytope can also be applied to solve the socalled post-office problem, see below.

The membership problem with witness is intermediate between membership and ray shooting. Rather surprisingly, the known data structures for the membership problem do not always provide a witness, and it seems nontrivial to find one. Finding a witness is essential in some applications of membership testing, see [MS].

In this paper we review some known data structures for the problem of membership in a convex polytope, and we add one new data structure, which is somewhat more efficient than the previously known ones. Our main goal is to extend known data structures for the membership problem, so that they are capable of handling the membership with withness and ray shooting.

The observation that ray shooting queries are somewhat related to point location and range searching is implicitly present in many papers. For instance, de Berg et al. $\left[\mathrm{dBH}^{+}\right]$developed sophisticated methods for ray shooting using range searching structures. Agarwal and Matoušek [AM2] observed that the transformation of a suitable range searching algorithm into an algorithm for ray shooting queries is possible under quite general conditions, if a sophisticated algorithmic technique, so-called parametric search (due to Megiddo [Me]), is

\footnotetext{
1 We say that a ray $\rho$ crosses a hyperplane $h$ if it intersects it but is not contained in it. Thus, it makes sense also to consider rays which go inside some face of $\mathscr{P}$, and we permit such rays.
} 
Table 1. Summary of data structures for ray shooting in a convex polytope ( $m$ is an adjustable parameter, $n \leq m \leq n^{(\mathrm{d} / 2\lrcorner}-$.

\begin{tabular}{ccc}
\hline $\begin{array}{c}\text { Space; } \\
\text { preprocessing }\end{array}$ & $\begin{array}{c}\text { Update time } \\
\text { (amortized) }\end{array}$ & $\begin{array}{c}\text { Ray shooting } \\
\text { queries }\end{array}$ \\
\hline$n ; n \log n$ & $\log ^{2} n$ & $n^{1-1 /\lfloor d / 2\rfloor+\delta}$ \\
$n^{\lfloor d / 2\rfloor+\delta}$ & $n^{\lfloor d / 2\lrcorner-1+\delta}$ & $\log n$ \\
$m^{1+\delta}$ & $\frac{m^{1+\delta}}{n}$ & $\frac{n}{m^{1 /\lfloor d / 2\rfloor}} \log n$ \\
$n ; n \log n$ & N/A & $n^{1-1 /\lfloor d / 2}(\log n)^{O(1)}$ \\
$m(\log n)^{O(1)}$ & N/A & $\frac{n}{m^{1 /\lfloor d / 2\rfloor}} \log n$ \\
$n^{\lfloor d i 2\rfloor}$ & N/A & $\log n$ \\
\hline$(\log n)^{\lfloor d / 2\rfloor-\delta}$ & & \\
\hline
\end{tabular}

employed. In particular, they obtained the first nontrivial algorithms for ray shooting in convex polytopes for an arbitrary dimension by this technique.

This application of parametric search makes the resulting ray shooting algorithm somewhat complicated, both conceptually and practically. Moreover, it typically increases the query time by a logarithmic factor.

We show that for some known data structures for the membership in a convex polytope, we can naturally pass to ray shooting without parametric search, only by a slight modification of the query answering algorithm and/or by augmenting the data structure. We then obtain the query time for ray shooting or witness finding exactly of the same order as for the membership problem.

Let us now review the known and new results for the considered problems in a quantitative form (see also Table 1). We distinguish static solutions (where the set of half-spaces defining the polytope $\mathscr{P}$ is fixed once and for all) and dynamic ones (where we allow insertions and deletions of half-spaces defining $\mathscr{P}$ ). As is the case with many problems of this type, slightly more efficient solutions are known for the static case than for the dynamic one.

For membership in a convex polytope, Clarkson [Cl1] gave a (static) solution with $O(\log n)$ query time, which uses $O\left(n^{\lfloor d / 2\rfloor+\delta}\right)$ space and expected preprocessing time. ${ }^{2}$ In [AM1] a dynamic counterpart of this data structure was given, with asymptotically the same space and query-time performance, and with $O\left(n^{\lfloor d / 2\rfloor-1+\delta}\right)$ amortized update time. ${ }^{3}$

\footnotetext{
${ }^{2}$ Throughout this paper, $\delta$ denotes an arbitrarily small positive constant. The multiplicative constants in the asymptotic bounds may depend on $\delta$.

${ }^{3}$ By saying that a data structure has $f(n)$ amortized update time we mean that starting with a current structure storing a set of $n$ hyperplanes, an arbitrary sequence of at most $n$ insertions and deletions can be performed in at most $n f(n)$ time.
} 
We describe a static data structure with $O(\log n)$ query time and with the space and preprocessing time requirement improved to $O\left(n^{\lfloor d / 2\rfloor} / \log ^{\lfloor d / 2\rfloor-\delta}\right)$. We apply the technique of bootstrapping developed by Chazelle and Friedman [CF] (they used it for point location among hyperplanes).

Why should such a small improvement be interesting? The worst-case combinatorial complexity of a polytope with $n$ facets is $\Theta\left(n^{\lfloor d / 2-}\right)$, and this is an intuitive reason to conjecture that, for membership queries with logarithmic time, the space requirements should be quite close to $n^{\lfloor d / 2\rfloor}$. A more daring form of this conjecture says that if $S(n)$ denotes the space and $Q(n)$ denotes the worst-case query time of a data structure for the membership problem, then $Q(n) \cdot S(n)^{1 /\llcorner d / 2\rfloor}=\Omega(n)$. Nothing even approaching a proof of this conjecture is known, but the work of Chazelle [Ch2] (which established a similar lower bound for simplex range searching, with $d$ replacing $\lfloor d / 2\rfloor$ ), and of Brönnimann and Chazelle [BC] (who proved a somewhat weaker lower bound for half-space range searching) give at least some ideological support to this conjecture. From this point of view, our algorithm would already be quite close to optimal, since it has $Q(n) S(n)^{1 / L d / 2\rfloor}=O\left(n(\log n)^{\delta}\right)$. Clarkson's solution gives $O\left(n^{1+\delta}\right)$ for this product, and another solution by Mulmuley [Mu] yields $O\left(n(\log n)^{c}\right)$ for a (not too small) constant $c$.

The previous paragraphs consider solutions to the membership problem with a logarithmic query time. At the other end of the spectrum, there is a solution by [Ma2], with $O(n)$ space, $O(n \log n)$ preprocessing time, and $\left.O\left(n^{1-1 / L d / 2}\right\rfloor(\log n)^{O(1)}\right)$ query time. A dynamic version with $O(n)$ space, $O\left(n^{1-1 /\lfloor d / 2\rfloor+\delta}\right)$ query time, and $O\left(\log ^{2} n\right)$ amortized update time is given in [AM1], as well as a dynamic version of Clarkson's data structure. Finally, the linear-space solutions can be easily combined with the previously mentioned large-space ones, obtaining a continuous tradeoff between space and query time, of the following form: Given a parameter $m, n \leq m \leq n^{\lfloor d / 2\rfloor}$, a static data structure can be built using $O\left(m(\log n)^{O(1)}\right)$ space and with $\left.O\left(\left(n / m^{1 /\lfloor d / 2}\right\rfloor\right) \log n\right)$ query time, or a dynamic data structure with $O\left(m^{1+\delta}\right)$ space and preprocessing time, $O\left(\left(n / m^{1 /\lfloor d / 2\rfloor}\right) \log n\right)$ query time, and $O\left(m^{1+\delta} / n\right)$ amortized update time.

Employing parametric search, any of the above-mentioned data structures for the membership problem can be used to answer ray shooting queries. The storage, preprocessing, and update time remain the same (as well as the whole data structure), but the query time increases by a polylogarithmic factor [AM2]. In this paper we obtain ray shooting algorithms for all these data structures, all of whose parameters (query time, storage, preprocessing time, and update time) are of the same order as the ones for the membership problem (given above). In particular, we can solve the membership problem with witness with these parameters. The bounds are summarized in Table 1 .

Note that by a well-known lifting transformation (see, e.g., [E]), nearest (and furthest) neighbor queries in dimension $d-1$ can be solved by shooting a vertical ray in the intersection of $n$ half-spaces in $\mathbb{E}^{d}$. We obtain a data structure for the post-office problem which is an improvement over previous results. Even in three-dimensional space, for instance, the best previous solution with space $O\left(n^{2}\right)$ had query time $O\left(\log ^{2} n\right)$, see $[\mathrm{Ch} 1]$ and $[\mathrm{AESW}]$. We improve the query time to $O(\log n)$. 
Let us mention some open problems. In our ray shooting algorithms we require that the query ray originates within the polytope $\mathscr{P}$ (which is the case in most applications). The general technique with parametric search can also handle rays originating outside $\mathscr{P}$ (see [AM2]); it would be interesting to extend our approach to this situation too.

The passage from membership queries to ray shooting can be viewed as giving one degree of freedom to the (originally fixed) query point, and performing one-dimensional optimization along the query ray. A natural further generalization considered in [MS] is to consider a query $k$-flat (for some $k>1$ ), and ask whether it intersects the polytope $\mathscr{P}$, or ask for the optimum of some linear function over the intersection of the query $k$-flat with $\mathscr{P}$. This problem can indeed be solved using a data structure for the membership problem. In [MS] this is done by a $k$-wise application of a multidimensional variant of parametric search, which produces a rather mysterious algorithm and quite large overhead in query time. It would thus be interesting to extend the methods of this present paper for also handling the queries with a $k$-flat and avoid the parametric search there.

Another problem to consider is the ray shooting in an arrangement of $n$ hyperplanes in $\mathbb{E}^{d}$. Applying parametric search to a suitable point location algorithm (e.g., that of Chazelle [Ch3]), $O\left(\log ^{2} n\right)$ query time with $O\left(n^{d}\right)$ space (or with $O\left(n^{d} / \log ^{d-\delta}\right)$ space, with some more tricks) is obtained. It would be interesting to get $O(\log n)$ query time with $O\left(n^{d}\right)$ or smaller space. This problem is solved in [CF] for vertical rays, but the solution does not extend to general ray shooting (see also [Ma3]).

\section{Algorithms with Almost Linear Space}

In this section it is more convenient to consider dual versions of the problems discussed in the introduction. Under a suitable duality transform, the set $H$ of hyperplanes extending the facets of the polytope $\mathscr{P}$ becomes a set $P$ of points. The membership problem then translates into the following query problem: given a query half-space $\gamma$, decide whether it is disjoint from $P$. The witness in the membership problem becomes simply a witness point of $P$ contained in $\gamma$. We call this the half-space emptiness problem.

We consider the linear-space data structures for the half-space emptiness problem of [Ma2] and its dynamization given in [AM1]. Neither of these data structures provides a witness directly. We show how to find one, and how to handle the (dual version of) ray shooting.

We start with several definitions. Let $P$ be an $n$-point set in $\mathbb{E}^{d}$. A simplicial partition of $P$ is a collection

$$
\Pi=\left\{\left(P_{1}, \Delta_{1}\right), \ldots,\left(P_{m}, \Delta_{m}\right)\right\}
$$

where the $P_{i}$ 's are nonempty sets (called the classes of $\Pi$ ) forming a partition of $P$ and each $\Delta_{i}$ is a relatively open simplex (not necessarily full-dimensional) containing $P_{i}$. The number $m$ is the size of the partition. 
The basis of the efficient algorithm for the half-space emptiness problem in [Ma2] is the so-called Partition Theorem for shallow hyperplanes. We say that a hyperplane $h$ is $k$-shallow (relative to $P$ ), if one of the half-spaces bounded by $h$ contains no more than $k$ points of $P$ in its interior. We say that a hyperplane $h$ crosses a simplex $\Delta$ if $\Delta \cap h \neq \varnothing$ but $\Delta \nsubseteq h$. For our purposes here, we use the following form of the theorem:

Theorem 1 [Ma2] (Partition Theorem for Shallow Hyperplanes). Let $P$ be a set of $n$ points in $\mathbb{E}^{d}, d \geq 3$, and let $r$ be a parameter, $1 \leq r \leq n / 2$. Then there exists a simplicial partition $\Pi$ of $P$ having size $O(r)$ with classes of size between $n / r$ and $2 n / r$, and such that any (n/r)-shallow hyperplane $h$ crosses at most $O\left(r^{1-1 /\lfloor d / 2\rfloor}+\log r\right)$ simplices of $\Pi$. For $r \leq n^{\alpha}$, where $\alpha>0$ is a certain constant (depending on the dimension $d)$, such a simplicial partition can be found in time $O(n \log r)$.

The preprocessing for a basic version of the half-space emptiness algorithm consists of building a partition tree using the above theorem. Each node $v$ of the tree corresponds to some subset $P_{v} \subseteq P$, the root corresponds to the whole set $P$. We choose a suitable large constant $r$, we find a simplicial partition of $P$ of size $O(r)$ as in the above theorem and we store the description of the simplices of this partition in the root of the tree. For every subset $P_{i}$ of this partition, we then build one subtree of the root in the same manner. The construction ends in the leaves of the tree, where the size of the subsets drops below some constant; then we explicitly store all the points of such a subset in the corresponding leaf.

The query answering algorithm deciding the emptiness of an open half-space $\gamma$ with a bounding hyperplane $h$ then uses the partition tree as follows: We start in the root of the tree, and we determine the simplices of the simplical partition stored there intersecting $\gamma$. If the number of such simplices is greater than the guaranteed maximal number $\kappa=O\left(r^{1-1 /\lfloor d / 2\rfloor}+\log r\right)$ of simplices crossed by any $(n / r)$-shallow hyperplane, then $\gamma$ is nonempty, in fact it contains at least $n / r$ points of $\boldsymbol{P}$ (since either there is a simplex completely contained in $\gamma$, or more than $\kappa$ simplices cross $h$, implying that $h$ is not $(n / r)$-shallow). Otherwise we proceed recursively down the tree into the at most $\kappa$ children of the current node for which the corresponding simplices intersect $\gamma$. This recursion terminates in leaves of the partition tree, where we simply check if any of the points stored there is contained in $\gamma$.

A straightforward analysis of this data structure shows that it requires $O(n)$ space, $O(n \log n)$ preprocessing time, and $O\left(n^{1-1 / L d / 2\rfloor+\delta}\right)$ query time, where $\delta>0$ tends to 0 with increasing $r$. A dynamic version of this data structure is described in [AM1]. First, it is shown that deletions of points from this data structure can be performed (rebuilding subtrees from which many points have been deleted). Insertions are then handled in a standard way (the "binary counting" pattern of Bentley and Saxe [BS]), using the decomposability of the half-space emptiness problem. The resulting (amortized) update time is $O\left(\log ^{2} n\right)$.

We now discuss several ways to find a witness using this data structure; later 
we generalize the methods to ray shooting also. The problematic situation is the following:

In the query answering algorithm we are at an inner node $v$, and we find that $\gamma$ intersects more than $\kappa$ simplices of the simplicial partition in $v$.

We say that such a half-space $\gamma$ is deep for $v$. By the argument above, we have $\left|\gamma \cap P_{v}\right|>n_{v} / r, n_{v}=\left|P_{v}\right|$, for any deep half-space $\gamma$. In the dynamic version of [AM1] the points get deleted from $P_{v}$, but after at most $n_{v} / 2 r$ deletions from $P_{v}$ the subtree rooted at $v$ is reconstructed from scratch, so in this case a deep half-space always contains at least $n_{v} / 2 r$ points of $P_{v}$. We describe three methods for finding a witness point of $P_{v}$ contained in a deep query half-space.

A Simple Randomized Algorithm. When we reach a node $v$ for which $\gamma$ is deep, we pick a random point $p$ in $P_{v}$. By the above, the probability that $p \in \gamma$ is at least $1 / 2 r$, which implies that the probability that we do not hit a point in $\gamma$ after $O(r \log n)$ trials is very small (less than $1 / n^{c}$, for a constant $c>0$ that can be made arbitrarily large). A random point in $P_{v}$ can be found in time $O\left(\log n_{v}\right)$ without having to store the set $P_{v}$ explicitly: if we store the number of points in $P_{v}$ with each node, we can just choose a random index in $\left\{1, \ldots, n_{v}\right\}$ and track down the point in the corresponding subtree, in $O\left(\log n_{v}\right)$ time. The time for witness finding is thus dominated by the total query time with high probability. We needed no auxiliary data structures (except for the point counts in nodes, whose maintenance is trivial), and so this query-answering algorithm can be used for the dynamic version as well.

A Deterministic Algorithm (Static Version). The key to a deterministic query algorithm providing a witness is the notion of $\varepsilon$-nets. A $(1 / r)$-net for an $n$-point set $P$ is a subset $R \subset P$ with the property that every half-space $\gamma$ with $|\gamma \cap P| \geq n / r$ contains a point of $R$. We use the following fact.

Fact 2. Let $P$ be a set of $n$ points in $\mathbb{E}^{d}, r \leq n$ a parameter. There exists a $(1 / r)$-net $R$ for $P$ with $|R|=O(r \log r)[\mathrm{HW}$, and it can be computed in time $O(n \log r)$ time if $r \leq n^{\alpha}$, where $\alpha>0$ is a certain constant, depending on the dimension [Ma1].

We augment every inner node $v$ of the partition tree as follows: We find a $(1 / r)$-net $R_{v}$ of size $O(r \log r)$ for $P_{v}$, and store it at $v$. When we reach a node $v$ in a query with $\gamma$ for which $\gamma$ is deep, we test all points $p \in R_{v}$ for containment in $\gamma$. Since $\gamma$ contains at least $n_{v} / r$ points of $P_{v}$, it must contain some point of $R_{v}$. Such a point, the witness, is thus found in additional time $O\left(\left|R_{v}\right|\right)=O(r \log r)$. Query time and storage of the structure thus remain the same as before, and this also holds for the preprocessing time (since $R_{v}$ can be computed in time linear in the size of $P_{v}$ ).

For a static data structure, the query time can be slightly improved, as in [Ma2]. We consider the case $d \geq 4$. Instead of setting $r$ to a large enough constant, we set $r=n_{v}^{\beta}$ in a node $v$, where $\beta>0$ is a suitable sufficiently small constant. 
Otherwise the data structure remains exactly the same. The space is still linear, the preprocessing time is $O(n \log n)$, and the query time is $O\left(n^{1-1 / \operatorname{ld} / 2}-(\log n)^{O(1)}\right)$, also when we count the additional witness finding time (this can be derived by analyzing simple recurrences, see [Ma2]). We do not know how to dynamize this improved data structure efficiently, so in the next algorithm we return to constant values of $r$ at every node.

A Deterministic Algorithm (Dynamic Version). In order to use the previous idea with $\varepsilon$-net in a dynamic setting, we need to maintain an $\varepsilon$-net dynamically. This is nontrivial, since if we delete a point belonging to the current $\varepsilon$-net, this $\varepsilon$-net has to be repaired somehow. Fortunately we can replace $\varepsilon$-nets by suitable simplicial partitions, which behave as "robust $\varepsilon$-nets" in some respect.

Above we have considered simplicial partitions for which every $k$-shallow hyperplane crosses possibly few simplices, for a suitable $k$. Here we need simplicial partitions for which any hyperplane crosses few simplices only. The following is shown in [Ma2]:

Lemma 3 [Ma2]. For every $n$-point set $P$ in $\mathbb{E}^{d}$ and a parameter $t(1<t \leq n / 2)$, there exists a simplicial partition $\Psi$ of size $O(t)$ for $P$, with class sizes between $n / t$ and $2 n / t$, and such that every hyperplane crosses at most $\xi=O\left(t^{1-1 / d}\right)$ simplices of $\Psi$. For $t$ bounded by a constant, such $a \Psi$ can be computed in time $O(n)$.

Let us consider such a simplicial partition $\Psi$ for $P$, and let $\gamma$ be an open half-space which contains none of the simplices of $\Psi$ completely. Since the bounding hyperplane of $\gamma$ crosses at most $\xi$ simplices of $\Psi$, all but $\xi$ simplices of $\Psi$ are disjoint from $\gamma$. Hence $\gamma$ contains at most $k=2 n \xi / t=O\left(n / t^{1 / d}\right)$ points of $P$. In other words, an open half-space containing more than $k$ points of $P$ completely contains some simplex of $\Psi$.

We now return to our partition tree and apply this as follows. In an inner node $v$, set $t=C r^{d}$, for a large enough constant $C$, and build an auxiliary simplicial partition $\Psi_{v}$ for the set $P_{v}$, as in Lemma 3. The constant $C$ is chosen in such a way that every open half-space $\gamma$ containing at least $n_{v} / 2 r$ points of $P_{v}$ completely contains some simplex of $\Psi_{v}$. The simplicial partition $\Psi_{v}$ will be our auxiliary data structure stored at $v$.

Suppose that we came to a node $v$ with a query half-space $\gamma$ which turned out to be deep for $v$ (so $\gamma$ contains some simplices of $\Psi$ ). Let $\gamma^{\prime} \subseteq \gamma$ be the open half-space whose boundary is parallel to the boundary of $\gamma$, and such that it does not contain the closure of any simplex of $\Psi_{v}$ but it contains some (relatively open) simplex $\Delta$ of $\Psi_{v}$. Thus, $\gamma^{\prime}$ arises by shifting $\gamma$ inward, until the boundary hits the last simplex of $\Psi_{v}$ remaining in the shifted half-space. Obviously $\gamma^{\prime}$ is nonempty, since it contains the points of $P_{v}$ in $\Delta$. On the other hand, $\left|P_{v} \cap \gamma^{\prime}\right|<n_{v} / 2 r$ : if it were not the case, then we might shift the (open) half-space $\gamma^{\prime}$ a little bit more, obtaining a half-space $\gamma^{\prime \prime}$ with $\gamma^{\prime \prime} \cap P_{V}=\gamma^{\prime} \cap P_{v}$ but such that no simplex of $\Psi_{v}$ is contained in $\gamma^{\prime \prime}$, which is impossible. 
Thus having a deep half-space $\gamma$ for a node $v$, we find $\gamma^{\prime}$ as above (in $O(t \log t)=O(1)$ time), and we continue the query with $\gamma^{\prime}$. This is contained in $\gamma$, intersects $P_{v}$ but it is not deep for $v$ anymore, so it intersects only

$$
\kappa=O\left(r^{1-1 /\lfloor d / 2\rfloor}+\log r\right)
$$

simplices of $\Pi_{v}$, and we can continue with the recursion in the appropriate children of $v$. Eventually we reach a leaf with a nonempty query half-space, and we find the desired withness there.

It remains to show how to maintain the simplicial partitions $\Psi_{v}$ under deletions of points. This is not difficult: Whenever at least $n_{v} / 2 t$ points of $P_{v}$ are deleted in a node $v$, we recompute the simplicial partition $\Psi_{v}$ anew, in $O\left(n_{v}\right)$ time. The amortized time is constant per deletion in one node, and $O(\log n)$ for the whole data structure. At the same time, these reconstructions guarantee that none of the simplices of $\Psi_{v}$ becomes empty, which is sufficient for a correct function of the algorithm. As for insertions, these need not be considered (since they are handled at a higher level of the dynamic data structure of [AM1]), but we could handle them easily anyway.

Note that we could also store the list of points of $P_{v}$ belonging to every simplex of the auxiliary simplicial partition $\Psi_{v}$, and thus find a withness for a deep half-space without further recursion. However, storing the lists of points in every node would raise the space requirements to $O(n \log n)$. Also, the above approach generalizes to ray shooting, as we now explain.

Ray Shooting Algorithms. For a point set $P$ and a nonvertical hyperplane $h$, let us call a point $p \in P$-extreme if there is no point of $P$ above the hyperplane passing through $p$ and parallel to $h$ (let us denote this hyperplane by $h(p)$ ). Let us consider the problem of finding an $h$-extreme point of $P$ for a query hyperplane $h$. This is the dual counterpart of the ray shooting problem with vertical rays emanating from $+\infty$.

It turns out that some of the data structures developed for half-space emptiness queries with witness can be used to find an $h$-extreme point quite easily. Let us consider the data structure for the static deterministic algorithm (the one with $\varepsilon$-nets). Given a query hyperplane $h$, we start in the root of the partition tree. In an inner node $v$ we proceed as follows: we find an $h$-extreme point $q$ of $R_{v}\left(R_{v}\right.$ is the $(1 / r)$-net at $v)$. Then we recursively find the $h$-extreme points for all children of $v$ corresponding to simplices of $\Pi_{v}$ intersecting the half-space above $h(q)$ (if there is no such simplex, then $q$ itself is $h$-extreme). Among these, we select an $h$-extreme one. In a leaf an $h$-extreme point is selected trivially. This algorithm is obviously correct, and the $(1 / r)$-net property of $R_{v}$ guarantees that $h(q)$ is an $n / r$-shallow hyperplane (relative to $P_{v}$ ). Hence we recurse in at most $\kappa$ children of $v$. Thus the running-time analysis is the same as for the basic half-space emptiness algorithm and we also get the same query time.

The $h$-extreme point problem can also be solved using the data structure for 
the dynamic deterministic algorithm (the one using auxiliary simplicial partitions). In fact, this is exactly what the above-described query-answering algorithm does, only we need to select the $h$-extreme point from all the leaves reached in the recursion.

As we pointed out, the $h$-extreme point problem is equivalent to vertical ray shooting from the inside of a convex polytope. The general ray shooting problem dualizes to the following problem: We are given a query (nonvertical) $(d-2)$-flat $a$, such that there is a hyperplane $h_{0}$ passing through $a$ and having no points of $P$ above it. We want to find an a-rotation extreme point of $P$, which is a point $p \in P$ such that there are no points of $P$ above the hyperplane passing through $p$ and $a$. The previously considered problem corresponds to the situation when $a$ is formally at infinity. An a-rotation extreme point can be found in almost the same way as we did for an $h$-extreme point; we leave details to the reader. Hence we can solve the ray shooting problem with the same complexity as the half-space emptiness problem.

We have thus given a new proof of the following result of [AM1] and [AM2]:

Theorem 4. The ray shooting problem for a convex polytope with $n$ facets in $\mathbb{E}^{d}$ can be solved with $O(n)$ space, $O(n \log n)$ preprocessing, $O\left(\log ^{2} n\right)$ amortized update time, and $O\left(n^{1-1 /\lfloor\mathrm{d} / 2\rfloor+\delta}\right)$ query time. For a static data strcture, the query time can be improved to $\left.O\left(n^{1-1 / L d / 2}\right\lrcorner(\log n)^{O(1)}\right)$.

\section{Ray Shooting with Logarithmic Query Time}

Another data structure for the polytope membership problem is a specialization of a result due to Clarkson [C12]; let us recall that it requires $O\left(n^{\lfloor d / 2\rfloor+\delta}\right)$ space and preprocessing time, and achieves $O(\log n)$ query time. A dynamic counterpart of this data structure was given in [AM1]. We explain a static data structure (similar to Clarkson's one) and the way we can find a witness point using this structure. Then we generalize to the ray shooting case.

We return to the primal setting of the problems, as discussed in the introduction. We may and do assume that the polytope $\mathscr{P}$ is the upper unbounded cell in an arrangement of a set $H$ of $n$ hyperplanes, i.e., the set of points lying above all hyperplanes of $H$. In this situation the membership problem takes the following special form: "Given a query point, determine whether it lies above all hyperplanes of $H . "$

Let $r \leq n$ be a parameter. For the sake of simplicity, we assume that the hyperplanes of $H$ are in general position. Let the level of a point $p \in \mathbb{E}^{d}$ with respect to $H$ be the number of hyperplanes $h \in H$ lying strictly above it. A collection $\Xi$ of simplices with disjoint interiors are called a $(1 / r)$-cutting for the $(\leq 0)$-level of $H$, provided that the simplices of $\Xi$ cover all points of level 0 (with respect to $H$, i.e., all points with no hyperplanes of $H$ lying strictly above them), and that each simplex of $\Xi$ is intersected by at most $n / r$ hyperplanes of $H$. We apply the following result: 
Theorem 5 (Shallow Cutting Lemma [Ma2]). Let $H, r$ be as above. Then there exists a $(1 / r)$-cutting $\Xi$ for the $(\leq 0)$-level of $H$, consisting of $\left.O\left(r^{\lfloor d / 2}\right\rfloor\right)$ simplices. For $r \leq n^{\alpha}$ (where $\alpha>0$ is a certain constant, dependent on the dimension), such a cutting can be computed in $O(n \log r)$ time.

For every simplex $\Delta \in \Xi$, we say that a hyperplane $h \in H$ is relevant for $\Delta$ if it lies above $\Delta$ or intersects $\Delta$. Let $H_{\Delta}$ denote the collection of hyperplanes relevant for $\Delta$.

A slightly modified Clarkson's structure for the membership problem is a tree-like structure, built recursively as follows: If the number of hyperplanes in $H$ is smaller than a suitable constant, then the list of hyperplanes of $H$ is simply stored; this will be a leaf node. A query is answered by testing the query point against each hyperplane of $H$.

If, on the other hand, $H$ is larger, a suitable parameter $r$ (a sufficiently large constant in Clarkson's original construction) is choosen, and a (1/r)-cutting $\Xi$ for the $(\leq 0)$-level of $H$, consisting of $O\left(r^{\lfloor d / 2\rfloor}\right)$ simplices, is computed. We store the cutting $\Xi$ in the root of the data structure, and, for every $\Delta \in \Xi$, we recursively build a subtree corresponding to the data structure for $H_{\Delta}$. The space $S(n)$ occupied by this data structure obeys the recursion

$$
S(n) \leq O\left(r^{\lfloor d / 2\rfloor}\right)+O\left(r^{\lfloor d / 2\rfloor}\right) S(n / r)
$$

which, for a sufficiently large but constant value of $r$, solves to $O\left(n^{\lfloor d / 2\rfloor+\delta}\right)$.

We now describe a query-answering algorithm finding a withness as well. A query with a point $q$ on $H$ is answered as follows: We begin at the root. Being in a nonleaf node $v$, we determine whether $q$ belongs to some simplex of $\Xi_{v}$, the cutting stored at $v$, and if there is such a simplex, we proceed recursively into the corresponding child of $v$. If there is no such simplex, it means that there is a hyperplane strictly above $q$.

In this situation we shoot a vertical ray $\rho$ from $q$ upward, we find the first simplex $\Delta$ of $\Xi_{v}$ intersected by $\rho$ and we recurse in the child corresponding to $\Delta$. We claim that some of the hyperplanes relevant for $\Delta$ must lie above $q$. Indeed, let us take the last hyperplane $h$ encountered along $\rho$ in the upward direction. The point $q \cap \rho$ has level 0 , and so it has to be contained in some simplex. Therefore $\Delta$ contains this point or some point below it, and so $h$ is relevant for $\Delta$, showing the correctness of this step.

In a leaf node we solve the query by inspecting all the hyperplanes in that node. This finishes the query algorithm. Since we spend a constant time in every node, the query time is $O(\log n)$.

The same method also works for the dynamic data structure of [AM1] and provides a witness hyperplane, while retaining the same $(O(\log n))$ query time. (The reader has to be familiar with the dynamic data structure in order to check our claim, since we do not explain the dynamic data structure. We apologize for withholding this information, but the data structure in question is technically fairly complicated.) Since the dynamic data structure requires a larger (nonconstant) value of $r$, we need some auxiliary data structure to find the first simplex of a 
cutting $\Xi$ crossed by the vertical ray $\rho$. This is done by point location in the projection of the cutting $\Xi$ on a horizontal hyperplane. The combinatorial complexity of this projection is bounded by a polynomial in the size of $\Xi$, and since this size is a very small fractional power of $n$, the complexity is still small compared with $n$. We can thus afford quite a wasteful approach: we extend every $(d-2)$-dimensional feature in this projection into a full hyperplane, and we use an algorithm for point location among hyperplanes with a logarithmic query time and a polynomial space (see, e.g., [CF]). This additional data structure does not increase the asymptotic space or query-time requirements.

Let us now turn to the ray shooting problem. We use the same data structure as for the membership problem, but with a slightly modified query algorithm. For the static case, assume we are given a query ray $\rho$ with origin $p$ lying above all hyperplanes in $H$. We begin the query answering at the root of the data structure. Being in a nonleaf node $v$, we determine the last simplex $\Delta$ of $\Xi_{v}$ intersected by $\rho$. Since $p$ lies above all hyperplanes of $H$, it is contained in a simplex of $\Xi_{v}$, hence such a last simplex $\Delta$ exists. We proceed recursively into the child of $v$ corresponding to $\Delta$. In a leaf node we simply test all hyperplanes to find the first one crossed by the ray $\rho$.

To prove the correctness of this algorithm, we have to show that the first hyperplane $h$ crossed by $\rho$ is relevant for $\Delta$. The argument is similar to the one employed above: Consider the point $\rho \cap h$. It has level 0 and must thus be contained in some simplex. Thus, $\Delta$ contains this point or a point on $\rho$ lying behind it (in the order along the ray $\rho$ ). Since $h$ contains the point $\rho \cap h, h$ must be relevant for $\Delta$.

In the dynamic data structure the size of the cuttings used is larger and we need a secondary point location structure to find the last simplex of $\Xi_{v}$ intersected by a query ray. It is sufficient to have a data structure with $O(\log s)$ query time and space and preprocessing time polynomial in $s$, the size of $\Xi$. This is discussed in the Appendix. We thus get

Theorem 6. The ray shooting problem for a convex polytope with $n$ facets in $\mathbb{E}^{d}$ can be solved with $O\left(n^{\lfloor d / 2\rfloor+\delta}\right)$ space and preprocessing time, $O(\log n)$ query time, and $O\left(n^{\lfloor d / 2\rfloor-1+\delta}\right)$ amortized update time.

\section{Reducing Storage for Static Data Structures with Logarithmic Query Time}

If we do not need a dynamic data structure, we can use the static data structure described in the previous section, but with the parameter $r$ set to $m^{\alpha}$ in an $m$-point node, where $\alpha>0$ is a suitable small constant. If $\alpha$ is chosen small enough, the storage requirement $S(n)$ follows the following recurrence: $S(n)=O(1)$ for $b \leq n_{0}=O(1)$, and

$$
\left.S(n)=c_{1} r^{\lfloor d / 2}\right\rfloor S(n / r)+c_{2} n^{\lfloor d / 2\rfloor}
$$

for some constants $c_{1}, c_{2}$. This solves to $S(n)=O\left(n^{\lfloor d / 2\rfloor} \log ^{c} n\right)$, where the constant 
$c$ depends on $c_{1}, c_{2}$. The query time follows a recursion of the form

$$
Q(n)=Q\left(n^{1-\alpha}\right)+O(\log n),
$$

which gives $Q(n)=O(\log n)$.

Let us now turn to the preprocessing time. The shallow cutting $\Xi$ can be constructed in time $O(n \log r)$ according to Theorem 5 . Identification of the sets $H_{\Delta}$ can trivially be done in time $\left.O\left(n r^{\lfloor/ 2}\right\rfloor\right)$ by testing every hyperplane with every simplex of the cutting.

The time to construct the secondary structure on $\Xi$ according to Lemma 11 (which is given in the Appendix) is polynomial in $r=n^{\alpha}$, and for sufficiently small $\alpha>0$ all this is bounded by $\left.O\left(n^{\lfloor d / 2}\right\rfloor\right)$ again. Thus we get the same recursion as for the space requirement and find that the proprocessing time is also bounded by $O\left(n^{\lfloor d / 2\rfloor} \log ^{c} n\right)$. We obtain

Lemma 7. The (static) ray shooting problem in a convex polytope with $n$ facets in $\mathbb{E}^{d}$ can be solved with preprocessing time and storage $\left.O\left(n^{\lfloor d / 2}\right\lrcorner(\log n)^{O(1)}\right)$ and with query time $O(\log n)$.

The partition tree data structures for ray shooting with linear space as in Section 2) can be combined with the data structures with logarithmic query time to obtain a continuous space-query-time tradeoff. Namely, we build only an upper part of the partition tree (as in the linear-space algorithm), stopping the recursive construction as soon as the size of the point set in a node drops below a suitably chosen number $s$, where $1<s<n$. For such a node $v$, instead of building the subtree for $P_{v}$, we store the data structure with logarithmic query time for $P_{v}$ at the node $v$. A similar construction appears in many previous papers (e.g., [CSW]), so we omit the details. A somewhat worse tradeoff for the ray shooting problem is given in [AM2]. We obtain the following:

Theorem 8. Given a parameter $m\left(n \leq m \leq n^{\lfloor d / 2} \downarrow\right)$, there is a dynamic data structure for ray shooting queries in a convex polytope with $n$ facets in $E^{d}$ with space and preprocessing time $O\left(m^{1+\delta}\right)$, amortized update time $O\left(m^{1+\delta} / n\right)$, and query time $\left.O\left(\left(n / m^{1 /\lfloor d / 2}\right\rfloor\right) \log n\right)$. For a static data structure, the space and preprocessing time can be improved to $O\left(m(\log n)^{O(1)}\right)$.

We now show that the idea of bootstrapping used by Chazelle and Friedman in $[\mathrm{CF}]$ can be used to improve the storage in Lemma 7 to $O\left(n^{\lfloor d / 2\rfloor} /\left(\log n^{\lfloor d / 2\rfloor-\delta}\right)\right.$. While [CF] needs four bootstrapping steps, we employ somewhat more powerful tools to obtain our result with a single such step. This idea could be used for the point location in hyperplane arrangements as well, and, in fact, the time bound given in $[\mathrm{CF}]$ can be slightly imporoved by following exactly the same ideas as presented here.

We need a lemma on random sampling due to Clarkson [Cl1], see also [CS]. For a collection $H$ of hyperplanes, let $\mathscr{U}(H)$ denote the set of all vertices of the upper envelope of $H$. 
Lemma 9. Let $H$ be a set of $n$ hyperplanes in $\mathbb{E}^{d}$ and let $\beta>0$ be a constant. For a random sample $R$ of $r$ hyperplanes from $H$, with probability at least $1 / 2$ the following is true: for all vertices $v \in \mathscr{U}(R)$, the number $n_{v}$ of hyperplanes $h \in H$ lying above $v$ is bounded by $O(n \log r / r)$, and the sum of $\sum_{v \in \mathscr{U}(R)} n_{v}^{\beta}$ is bounded by $O\left(r^{\lfloor d / 2\rfloor}(n / r)^{\beta}\right)$.

The improved structure can now be derived as follows: We take a random sample $R$ of $H$ of size $r=n / \log ^{k} n$ for some parameter $k$ (which is determined later), and build a structure according to Lemma 7 for ray shooting in the upper cell of the arrangement of $R$. Furthermore, for every vertex $v$ of the upper envelope of $R$ we identify the set $H_{v}$ of hyperplanes $h \in H$ lying above $v$. Let $n_{v}=\left|H_{v}\right|$.

We then construct, for every such vertex $v$, a secondary structure as in Theorem 8 with storage $s=O\left(n_{v}^{\beta}\left(\log n_{v}\right)^{\gamma}\right)(\gamma$ a constant $)$ and query time $O\left(n_{v}^{1-\beta /\lfloor d / 2\lrcorner}\right)$ $\left(1 \leq \beta<\lfloor d / 2\rfloor\right.$ is yet another constant to be chosen later ${ }^{4}$. By Lemma 9 we can choose $R$ such that $n_{v}=O\left(\log ^{k+1} n\right)$ for all vertices $v \in \mathscr{U}(R)$, and at the same time the total storage for the secondary structures is

$$
\begin{aligned}
\sum_{v \in \mathscr{\mathscr { U }}(R)} n_{v}^{\beta}\left(\log n_{v}\right)^{\gamma} & =O\left(r^{\lfloor d / 2\rfloor}(n / r)^{\beta}(\log \log n)^{\gamma}\right) \\
& =O\left(n^{\lfloor d / 2\rfloor}(\log n)^{k \beta-k\lfloor d / 2\rfloor}(\log \log n)^{\gamma}\right) .
\end{aligned}
$$

Consider now a query with a ray $\rho$ with origin $p$ above the upper envelope of $H$. Let $h$ be the first hyperplane in $R$ crossed by $\rho$, and let $q=\rho \cap h$. We determine a set $V$ of vertices of the upper envelope of $R$ such that $q$ lies in the convex hull of $V$. This can be done by repeated ray shooting queries in the upper cell of the arrangement of $R$ : We first find $q$ and $h$ by shooting with the ray $\rho$. Then take an arbitrary vertex $p_{1}$ of the upper envelope of $R$ which is incident to $h$, and shoot with a ray $\rho_{1}$ with origin $p_{1}$ and going through $q$. This ray lies in $h$. Let $h_{1}$ be the first hyperplane in $R$ crossed by $\rho_{1}$, and let $q_{1}$ be the point of intersection. Take an arbitrary point $p_{2}$ incident to $h$ and $h_{1}$. Shoot again with the ray $\rho_{2}$ with origin $p_{2}$ and going through $q_{1}$, finding a new hyperplane $h_{2} \in R$ and a point $q_{2}$. In this fashion we proceed until the intersection of $h \cap h_{1} \cap h_{2} \cap \cdots \cap h_{i}$ is a vertex $p_{i+1}$. Then we can take $V=\left\{p_{1}, p_{2}, \ldots, p_{i+1}\right\}$. The size of $V$ is at most $d$.

We now query the secondary structures associated with the at most $d$ vertices in $V$ with the ray $\rho$, and output the first hyperplane crossed in any of the subproblems.

It remains to show that the first hyperplane $h^{\prime}$ in $H$ crossed by $\rho$ appears in $H_{v}$ for some vertex $v \in V$. If the solution hyperplane $h^{\prime}$ is not identical to $h$, it must lie above the point $q$ where $\rho$ intersects $h$. Since $q$ lies in the convex hull of the $v \in V$, $h^{\prime}$ lies above at least one of the vertices $v$.

Since $n_{v}=O\left(\log ^{k+1} n\right)$, the query time of our combined structure is bounded by

$$
O\left(\log n+(\log n)^{(k+1)(1-\beta / L d / 2 \downarrow)}\right)
$$

\footnotetext{
${ }^{4}$ A weaker form of the tradeoff with storage $O\left(m^{1+\delta}\right)$ would suffice, such as in [AM2]
} 
We choose $\beta=\lfloor d / 2\rfloor(k /(k+1))$. This implies that the query time is bounded by $O(\log n)$. The storage for the primary data structure for ray shooting in the upper cell of the arrangement of $R$ is (by Lemma 7) $\left.O\left(r^{L d / 2}\right\lrcorner(\log n)^{c_{1}}\right)$ for some constant $c_{1}$. For $k$ large enough, this will not exceed $O\left((n / \log n)^{\lfloor d / 2\rfloor}\right)$. The storage for the secondary structures is bounded by

$$
O\left(\frac{n^{\lfloor d / 2\rfloor}}{(\log n)^{\lfloor d / 2\rfloor-\lfloor d / 2\rfloor /(k+1)}}(\log \log n)^{\gamma}\right),
$$

as we find from (1) and the choice of $\beta$. Choosing $k$ large enough we get the total storage of order $O\left(n^{\lfloor d / 2\rfloor} /(\log n)^{\lfloor d / 2\rfloor-\delta}\right)$.

Let us turn to the cost of preprocessing. The problem is to find a sample $R$ which conforms to the requirements of Lemma 9. Those can be checked once we know the number of hyperplanes in the lists $H_{v}$ for every vertex $v \in \mathcal{U}(R)$ (these lists have to be computed anyway). We just take a random sample $R$ of the appropriate size, and construct its upper envelope $\mathscr{U}(R)$. Then we sequentially take all $n$ hyperplanes $h$ from $H$ and find all $v \in \mathscr{U}(R)$ which lie below $h$. It is well known that this can be done in time linear in the number of such vertices once one such vertex is known (see, e.g., [CS]). On the other hand, we get such a vertex by linear programming on $R$ in time $O(r)$ (see [S]), so the total time for this step is $O(n r)$ plus the total number of vertices found. We stop this algorithm if it tries to report more than $O\left(n r^{\lfloor d / 2\rfloor-1}\right)$ such vertices (because then the sample $R$ is bad), otherwise we check whether it fulfills the requirements of Lemma 9. If not, we discard $R$ and take a new random sample. By Lemma 9 , the expected number of such trials is constant, so we can find $R$ in expected time $O\left(n r^{\lfloor d / 2\rfloor-1}\right)$ (and identify the lists $H_{v}$ in the same time). The secondary structures can be computed in time linear in their storage, so again we have the same recursion as for the space. We have thus proven

Theorem 10. The (static) ray shooting problem in a convex polytope with $n$ facets in $\mathbb{E}^{d}$ can be solved with preprocessing time and storage $O\left(n^{\lfloor d / 2\rfloor} /(\log n)^{\lfloor/ 2\rfloor-\delta}\right)$ and with query time $O(\log n)$.

\section{Acknowledgments}

Many thanks go to Emo Welzl for lots of helpful discussions. We would also like to thank Pankaj Agarwal for his comments on previous versions of this work. Furthermore, we are grateful to the anonymous referees for their constructive criticism.

\section{Appendix. Finding the Last Simplex Intersected by a Query Ray}

Here we treat the problem arising in Section 3: given a set of $s$ simplices in $\mathbb{E}^{d}$ with disjoint interiors, preprocess it in polynomial time and space so that, given a query ray $\rho$, the last simplex intersected by $\rho$ can be detected in $O(\log s)$ time. 
Lemma 11. Given a set $\Xi$ of $s$ simplices with disjoint interiors in $\mathbb{E}^{d}$. We can build in time $O\left(s^{8 d+2}\right)$ a data structure of size $O\left(s^{8 d-6+\delta}\right)$ that permits queries of the form "Given a query ray $\rho$, find the last simplex $\Delta$ in $\Xi$ intersected by $\rho$ " with query time $O(\log s)$.

Proof. We apply a standard locus approach. We consider the space of all rays. The loci corresponding to rays with the same last simplex induce a subdivision of this space. We can do point location in this subdivision using the following result by Chazelle et al. [CEGS].

Lemma 12 [CEGS]. Let $f_{1}, \ldots, f_{N}$ be $D$-variate polynomials of degrees bounded by a constant ( $D$ is also considered a constant). For a $D$-dimensional variable $x:=\left(x_{1}, x_{2}, \ldots, x_{D}\right)$, let $\operatorname{sign}_{i}(x)$ be the sign of $f_{i}(x)$, and

$$
\operatorname{sign}(x):=\left(\operatorname{sign}_{1}(x), \ldots, \operatorname{sign}_{N}(x)\right)
$$

be the sign vector of $x$ with respect to the family $\left(f_{i}\right)$. Assume we are given a label for every possible value of sign $(x)$.

There is a data structure with storage and preprocessing time $O\left(N^{2 D-3+\delta}\right)$ which, given a $D$-dimensional vector $x$, finds the label associated with sign $(x)$ in time $O(\log N)$.

This result is applied as follows. We represent the query ray $\rho$ by its origin $p$ and by a direction vector $u$. We thus have a $D:=2 d$ dimensional $^{5}$ representation for $\rho$. Consider now a fixed simplex $\Delta$. We compute a constant number of polynomials in the $D=2 d$ variables representing $\rho$ to test whether $\rho$ intersects $\Delta$. We first observe that $\rho$ intersects $\Delta$ if either the origin $p$ of $\rho$ lies in $\Delta$, or if some facet $f$ of $\Delta$ is visible from $p$ and the direction $u$ lies in the convex cone with apex $p$ spanned by $f$. The first case can easily be tested by $d+1$ linear inequalities (polynomials of degree one). For the second case we first identify the facets $f$ visible from $p$ using the same inequalities as in the first case.

We now have a facet $f$ of $\Delta$ visible from $p$ and wish to test whether $u$ lies in the cone spanned by $f$. This can be done by checking the orientation of $u$ with respect to the $d$ hyperplanes that pass through $p$ and the $d$ ridges $((d-2)$-faces) of $f$. For every ridge, we choose $d-1$ affinely independent points $q_{1}, q_{2}, \ldots, q_{d-1}$. The orientation of $u$ with respect to the hyperplane spanned by $p$ and $q_{1}$, $q_{2}, \ldots, q_{d-1}$ can be tested by computing the orientation of the simplex spanned by $u$ and the $d-1$ vectors $q_{1}-p, q_{2}-p, \ldots, q_{d-1}-p$. This orientation can be found by computing the determinant of the matrix whose rows are these $d$ vectors, which corresponds to the evaluation of a polynomial of degree $d$.

In this fashion we have determined a set of $O(s)$ polynomials. The signs of these polynomials for the variable representing a given ray $\rho$ give us complete information as to which simplices are intersected by $\rho$, and, if a simplex $\Delta$ is intersected, through which facet $f$ of $\Delta$ the ray $\rho$ enters the simplex $\Delta$. It remains to determine the last simplex intersected by $\rho$.

\footnotetext{
${ }^{5}$ This is admittedly not optimal.
} 
To this end we add another set of $O\left(s^{2}\right)$ polynomials. Assume that $\rho$ intersects two simplies $\Delta_{1}$ and $\Delta_{2}$, and enters them through their facets $f_{1}$ and $f_{2}$, respectively. Let $h_{1}$ and $h_{2}$ be the hyperplanes containing $f_{1}$ and $f_{2}$. We can then test which of the two simplices is intersected first by testing which of the two intersections of $\rho$ with $h_{1}$ and $h_{2}$ lies closer to $p$. However, this can again be tested by determining the orientation of $u$ with respect to the hyperplane through $p$ and the $(d-2)$-flat $h_{1} \cap h_{2}$. This can be dealt with as above. In this fashion we can add a polynomial for every pair of facets of different simplices.

The sign sequence of the resulting set of polynomials uniquely determines which simplex is the last one intersected by $\rho$. We can therefore appeal to Lemma 12 , with parameters $N \in O\left(s^{2}\right)$ and $D=2 d$, to obtain a search structure with the time bounds claimed above. Given a ray $\rho$, this structure returns the label of the last simplex $\Delta$ intersected by $\rho$.

\section{References}

[AESW] P. K. Agarwal, H. Edelsbrunner, O. Schwarzkopf, and E. Welzl. Euclidean minimum spanning trees and bichromatic closest pairs. Discrete \& Computational Geometry, 6:407-422, 1991.

[AM1] P. K. Agarwal and J. Matoušek. Dynamic half-space range reporting and its applications. Technical Report CS-1991-43, Duke University, 1991.

[AM2] P. K. Agarwal and J. Matoušek. Ray shooting and parametric search. In Proc. 24th ACM Symposium on Theory of Computing, pages 517-526, 1992. Full version as Technical Report CS-1991-22, Duke University, 1991.

[BS] J. L. Bentley and J. B. Saxe. Decomposable searching problems I: Static-to-dynamic transformation. Journal of Algorithms, 1:301-358, 1980.

[BC] H. Brönnimann and B. Chazelle. How hard is halfspace range searching? In Proc. 8th Symposium on Computational Geometry, pages 271-275, 1992.

[CEGS] B. Chazelle, H. Edelsbrunner, L. Guibas, and M. Sharir. A singly-exponential stratification scheme for real semi-algebraic varieties and its applications. In Proc. 16th International Colloquium on Automata, Languages and Programming, pages 179-192. Lecture Notes in Computer Science, vol. 372. Springer-Verlag, Berlin, 1989.

[CF] B. Chazelle and J. Friedman. Point location among hyperplanes and vertical ray shooting. Computational Geometry: Theory and Applications (to appear).

[Ch1] B. Chazelle. How to search in history. Information and Control, 64:77-99, 1985.

[Ch2] B. Chazelle. Lower bounds on the complexity of polytope range searching. Journal of the American Mathematical Society, 2(4):637-666, 1989.

[Ch3] B. Chazelle. Cutting hyperplanes for divide-and-conquer. Discrete \& Computational Geometry, 9:145-158, 1993.

[C11] K. L. Clarkson. New applications of random sampling in computational geometry. Discrete \& Computational Geometry, 2:195-222, 1987.

[Cl2] K. L. Clarkson. A randomized algorithm for closest-point queries. SIAM Journal on Computing, 17:830-847, 1988.

[CS] K. L. Clarkson and P. W. Shor. Applications of random sampling in computational geometry, II. Discrete \& Computational Geometry, 4:387-421, 1989.

[CSW] B. Chazelle, M. Sharir, and E. Welzl. Quasi-optimal upper bounds for simplex range searching and new zone theorems. Algorithmica, 8:407-429, 1992.

$\left[\mathrm{dBH}^{+}\right] \mathrm{M}$. de Berg, D. Halperin, M. Overmars, J. Snoeyink, and M. van Kreveld. Efficient fay shooting and hidden surface removal. Algorithmica (to appear).

[E] H. Edelsbrunner. Algorithms in Combinatorial Geometry. Springer-Verlag, New York, 1987. 
[HW] D. Haussler and E. Welzl. $\varepsilon$-nets and simplex range queries. Discrete \& Computational Geometry, 2:127-151, 1987.

[Ma1] J. Matoušek. Efficent partition trees. Discrete \& Computational Geometry, 8:315-334, 1992.

[Ma2] J. Matoušek. Reporting points in halfspaces. Computational Geometry: Theory \& Applications, 2:169-186, 1992.

[Ma3] J. Matoušek. On vertical ray shooting in arrangements. Computational Geometry: Theory \& Applications (to appear).

[Me] N. Megiddo. Applying parallel computation algorithms in the design of serial algorithms. Journal of the $A C M, 30: 852-865,1983$.

[MS] J. Matoušek and O. Schwarzkopf. Linear optimization queries. In Proc. 8th ACM Symposium on' Computational Geometry, pages 16-25, 1992.

[Mu] K. Mulmuley. Randomized multidimensional search trees: further results in dynamic sampling. In Proc. 32nd Symposium on Foundations of Computer Science, pages 216-227, 1991.

[S] R. Seidel. Low dimensional linear programming and convex hulls made easy. Discrete \& Computational Geometry, 6(5):423-434, 1991.

Received May 6, 1992, and in revised form January 29, 1993. 